\title{
Influence of Strain Ratio on Bending Fatigue Life and Fatigue Crack Growth in TiNi Shape-Memory Alloy Thin Wires
}

\author{
Ryosuke Matsui $^{1, * 1}$, Yoshiyasu Makino ${ }^{2, * 2}$, Hisaaki Tobushi ${ }^{2, * 3}$, \\ Yuji Furuichi ${ }^{2}$ and Fusahito Yoshida ${ }^{1}$ \\ ${ }^{1}$ Department of Mechanical System Engineering, Hiroshima University, Higashi-Hiroshima 739-8511, Japan \\ ${ }^{2}$ Department of Mechanical Engineering, Aichi institute of Technology, Toyota 470-0392, Japan
}

\begin{abstract}
The influence of strain ratio on bending fatigue properties of TiNi shape-memory alloy thin wires and the process of fatigue crack propagation were investigated. The results obtained are summarized as follows. (1) The martensitic transformation stress of a superelastic thin wire is higher than that of a shape memory wire, resulting in shorter fatigue life of the superelastic wire. The maximum bending strain of fatigue limit is the martensitic-transformation starting strain. (2) The plane-bending fatigue life curve is expressed by a power function of maximum strain $\varepsilon_{\max }$ and the number of cycles to failure. The smaller the strain ratio, the shorter the fatigue life. (3) In both rotating bending and plane bending, fatigue cracks nucleate on the surface of the wire. One fatigue crack grows preferentially and the fatigue-crack propagated region of fracture surface is fan-shaped. (4) If $\varepsilon_{\max }$ is larger than $1 \%, \varepsilon_{\max }$ during the rotating-bending fatigue test becomes a little smaller than that of the initial value. (5) The fatigue crack length can be estimated by measuring increase in electric resistance based on decrease in cross-sectional area due to fatigue crack propagation. (6) The fatigue crack length of the notched wire is expressed by a power function of the number of cycles.
\end{abstract}

(Received October 7, 2005; Accepted December 15, 2005; Published March 15, 2006)

Keywords: shape memory alloy, fatigue, bending, superelasticity, strain ratio, crack propagation

\section{Introduction}

Shape memory alloy (SMA) is expected to be applied as intelligent materials since it shows the unique characteristics of the shape memory effect (SME) and superelasicity (SE). ${ }^{1-5)}$ Most SMA elements with using these characteristics perform cyclic motions. In these cases, fatigue of SMA is one of the important properties in view of evaluating functional characteristics as SMA elements. ${ }^{6-8)}$ Fatigue properties of SME and SE are complex since they depend on stress, strain, temperature and time which are related to the martensitic transformation (MT). In order to clarify the fatigue properties of SMA, it is necessary to investigate the influence of these factors on fatigue properties. Although many investigations on fatigue of SMA have been carried out till now, the bending fatigue properties of SMA thin wires practically used are not investigated enough. ${ }^{9-13)}$ The bending fatigue properties of SMA thin wires have been investigated mainly on rotating bending till now. However, most SMA elements are subjected to plane bending in practical applications. Therefore, it is important to clarify the fatigue properties not only due to rotating bending but also due to plane bending.

In the present study, the fatigue properties of TiNi SMA thin wires which show SME and SE at room temperature were investigated with respect to cyclic bending deformation. In the fatigue tests, plane bending and rotating bending were carried out. The fatigue properties of both wires, the influence of strain ratio on fatigue life and the fatigue crack growth were discussed.

\footnotetext{
${ }^{* 1}$ Graduate Student, Hiroshima University

${ }^{*}$ Graduate Student, Aichi Institute of Technology

${ }^{* 3}$ Corresponding author, E-mail: tobushi@aitech.ac.jp
}

\section{Experimental Method}

\subsection{Materials and specimen}

The materials used were TiNi SMA thin wires made by Furukawa Electric Co.: a wire (SME-NT) and a wire (SE-NT) which showed SME and SE at room temperature, respectively. The diameters of SME-NT and SE-NT were 0.75 and $0.5 \mathrm{~mm}$, respectively. Both wires were shape-memorized with a rectilinear shape. A shorter specimen with a length of 60-80 $\mathrm{mm}$ was chosen to give a larger bending strain.

\subsection{Experimental apparatus}

In the fatigue tests, a pulsating-plane bending fatigue test machine, ${ }^{10)}$ an alternating-plane bending fatigue test machine, ${ }^{11)}$ and a rotating-bending fatigue test machine ${ }^{9)}$ were used. In the tests, the number of cycles to failure was obtained under a certain values of bending strain, temperature and frequency. A scanning electron microscopy (SEM) was used to observe the fracture surface of the specimen.

\subsection{Experimental procedure}

The fatigue tests were carried out at room temperature in air. In the bending fatigue tests, maximum bending strain $\varepsilon_{\max }$ and minimum bending strain $\varepsilon_{\min }$ on the surface of the specimen in each cycle were designated.

\section{Experimental Results and Discussion}

\subsection{Fatigue life of SME-NT and SE-NT}

Figure 1 shows the relationships between strain amplitude $\varepsilon_{\mathrm{a}}$ and the number of cycles to failure $N_{\mathrm{f}}$ obtained by a perfect pulsating-plane bending fatigue test for SME-NT and SE-NT. As can be seen, the slopes of both strain-life curves are steep in the low-cycle fatigue region. The corners of the curves appear in the vicinity of $\varepsilon_{\mathrm{a}}=0.5 \%$ and $N_{\mathrm{f}}=4 \times 10^{5}$ cycles for SME-NT and in the vicinity of $\varepsilon_{\mathrm{a}}=0.35 \%$ and $N_{\mathrm{f}}=$ 


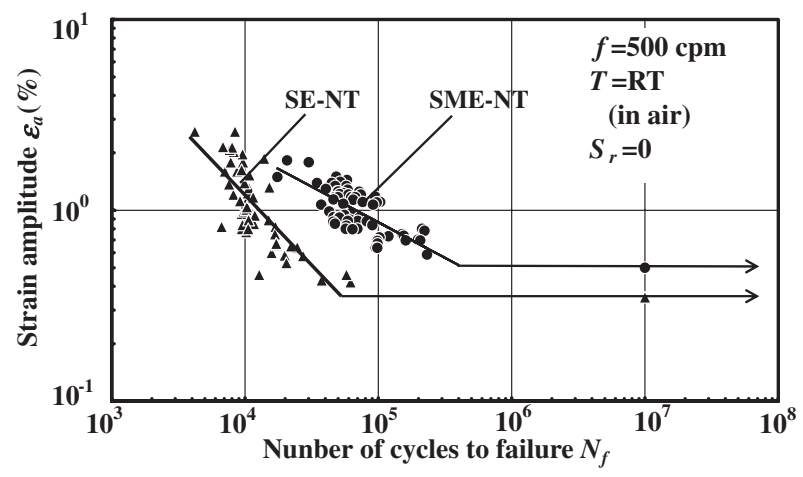

Fig. 1 Fatigue life curves for pulsating-plane bending.

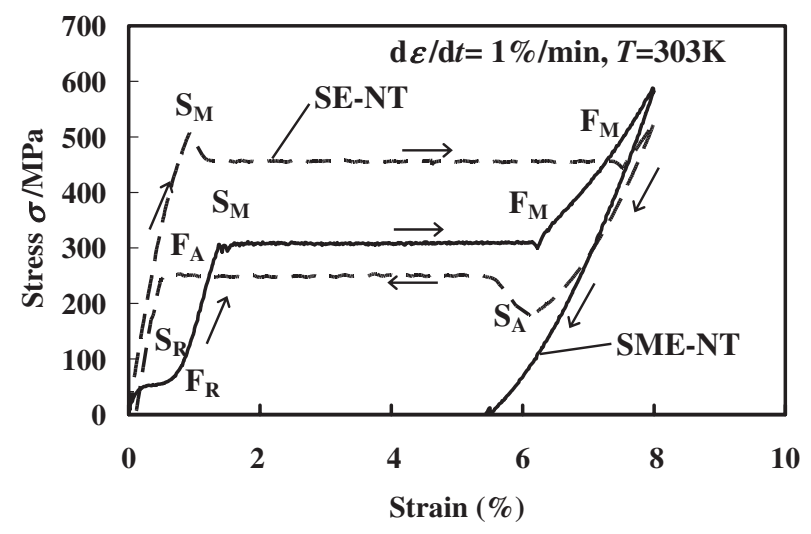

Fig. 2 Stress-strain curves.

$5 \times 10^{4}$ cycles for SE-NT, respectively. The strain amplitude for a plateau of the curve must correspond to the fatigue limit. The relationships in the shorter life side than the corner are expressed by the straight lines on the logarithmic graph. There is a large difference between fatigue lives of both materials in the low-cycle fatigue region.

In order to discuss the difference in the low-cycle fatigue region, the stress-strain curves of both materials are shown in Fig. 2. As can be seen, the MT stress $\sigma_{\mathrm{M}}$ shown by the stress plateau is $300 \mathrm{MPa}$ for SME-NT and $450 \mathrm{MPa}$ for SE-NT, respectively. The MT stress in SE-NT is higher than that in SME-NT and therefore fatigue damage is larger, resulting in shorter fatigue life in the range of small strain amplitude.

On the other hand, fatigue life of SME-NT becomes short markedly as $\varepsilon_{\max }$ increases, and therefore the difference of fatigue life between both materials decreases. In order to discuss this phenomena, Fig. 3 shows the schematic stressstrain diagrams of surface elements of both wires for maximum strain $\varepsilon_{\max }=2$ and $4 \%$ in each cycle during the fatigue test. In Fig. 3, it is assumed that the MT stress $\sigma_{M}$ is equal in compression and in tension. As can be seen in Fig. 3, the area surrounded by the hysteresis loop in each cycle denotes the dissipated work $W_{\mathrm{d}}$ per unit volume. $W_{\mathrm{d}}$ for SENT and SME-NT are expressed as follows, respectively.

$$
\begin{array}{ll}
W_{\mathrm{d}}=\left(\sigma_{\mathrm{M}}-\sigma_{\mathrm{A}}\right)\left(\varepsilon_{\max }-\varepsilon_{\mathrm{MS}}\right) & : \text { SE-NT } \\
W_{\mathrm{d}}=2 \sigma_{\mathrm{M}}\left(\varepsilon_{\max }-2 \sigma_{\mathrm{M}} / E\right) & : \text { SME-NT }
\end{array}
$$

where $\sigma_{\mathrm{A}}, \varepsilon_{\mathrm{MS}}$ and $E$ denote reverse transformation stress,

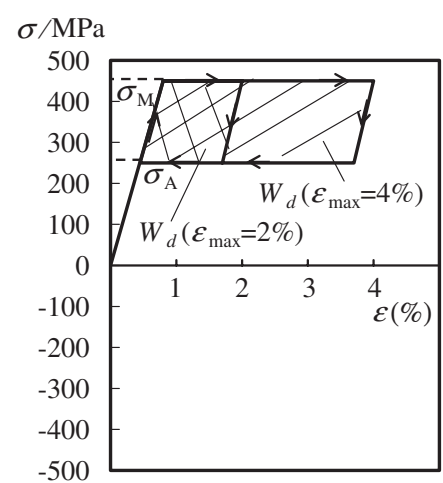

(a) SE-NT

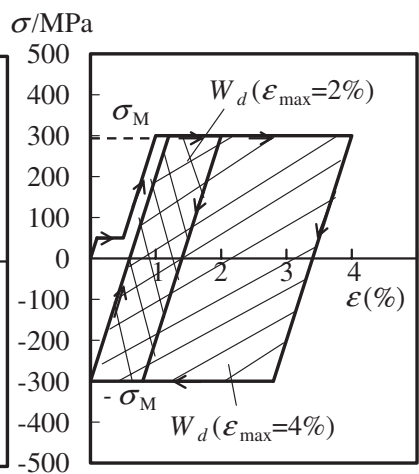

(b) SME-NT
Fig. 3 Schematic stress-strain diagrams for SE-NT and SME-NT in pulsating bending $\left(S_{\mathrm{r}}=0\right)$.

MT starting strain and elastic modulus, respectively. In the case of SE-NT, $W_{\mathrm{d}}=2.6 \mathrm{MJ} / \mathrm{m}^{3}$ for $\varepsilon_{\max }=2 \%$ and $W_{\mathrm{d}}=$ $6.6 \mathrm{MJ} / \mathrm{m}^{3}$ for $\varepsilon_{\max }=4 \%$. In the case of SME-NT, $W_{\mathrm{d}}=1.8$ $\mathrm{MJ} / \mathrm{m}^{3}$ for $\varepsilon_{\max }=2 \%$ and $W_{\mathrm{d}}=13.8 \mathrm{MJ} / \mathrm{m}^{3}$ for $\varepsilon_{\max }=4 \%$. Therefore, $W_{\mathrm{d}}$ of SME-NT increases markedly as $\varepsilon_{\max }$ increases. With respect to fatigue life for both materials, the influence of $\sigma_{\mathrm{M}}$ on fatigue damage is large in the range of small $\varepsilon_{\max }$, resulting in short fatigue life for SE-NT. On the other hand, since $W_{\mathrm{d}}$ for SME-NT becomes very large as $\varepsilon_{\max }$ increases, the influence of $W_{\mathrm{d}}$ on fatigue damage becomes significant, resulting in short fatigue life. Therefore, the difference of fatigue life between both materials must become small as $\varepsilon_{\max }$ increases based on the influence of $W_{\mathrm{d}}$.

Based on the stress-strain curves shown in Fig. 2, the strain corresponding to the fatigue limit can be considered as follows. In the perfect pulsating-plane bending fatigue test, strain amplitude $\varepsilon_{\mathrm{a}}$ is a half of maximum strain $\varepsilon_{\max }$. Therefore, the maximum strain $\varepsilon_{\max }$ at the fatigue limit is $1 \%$ for SME-NT and $0.7 \%$ for SE-NT, respectively. The strain of $1 \%$ for SME-NT corresponds to the region after the R-phase transformation finishing point $\mathrm{F}_{\mathrm{R}}$ and that of $0.7 \%$ for SE-NT corresponds to the vicinity of after the start of the MT starting point $\mathrm{S}_{\mathrm{M}}$. Therefore, the strain regions necessary to be considered in the low-cycle fatigue exist after the finish of the R-phase transformation for SME-NT and after the start of the MT for SE-NT, respectively.

\subsection{Influence of strain ratio on fatigue life for SE-NT}

Figure 4 shows the relationships between maximum strain $\varepsilon_{\max }$ and the number of cycles to failure $N_{\mathrm{f}}$ for SE-NT obtained by the plane-bending fatigue test with various strain ratios $S_{\mathrm{r}}$. In the figure, the points with arrows at $10^{6}$ and $10^{7}$ cycles denote cases where failure did not occur. As can be seen, the slopes of the strain-life curves are steep in the lowcycle fatigue region. Maximum strain at the corner of the curves is about $0.7 \%$. Although it is necessary to discuss the influence of $S_{\mathrm{r}}$ on the fatigue limit in more detail, maximum strain of the fatigue limit is close to the MT starting strain of $0.7 \%$ for every $S_{\mathrm{r}}$.

In order to observe the low-cycle fatigue properties in detail, the low-cycle fatigue curves are enlarged in Fig. 5. As can be seen, the relationships between maximum strain $\varepsilon_{\max }$ 


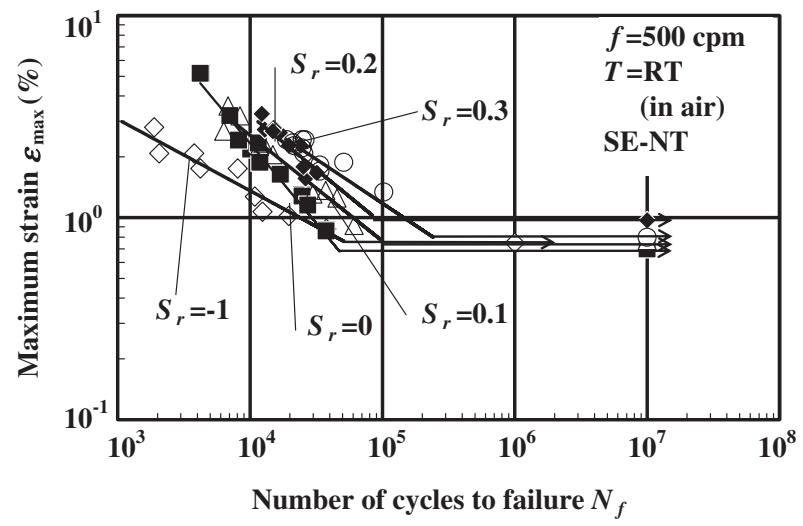

Fig. 4 Fatigue life curves for various strain ratios $S_{\mathrm{r}}$ in plane bending.

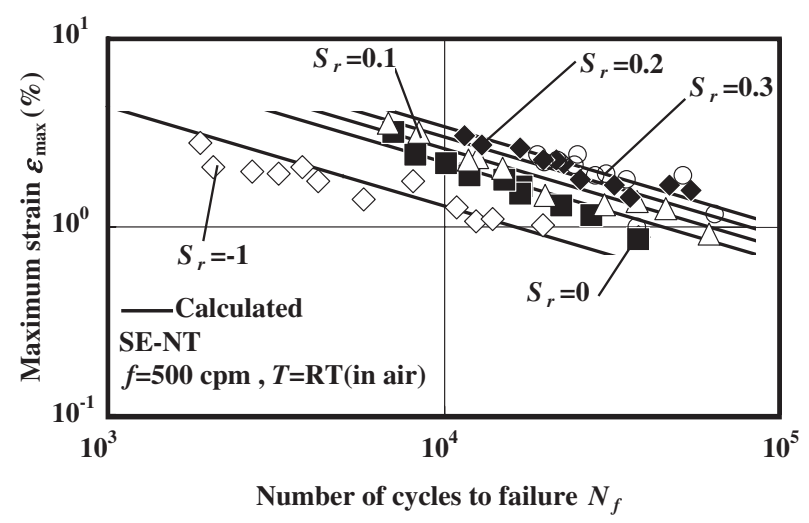

Fig. 5 Low-cycle fatigue life curves for various strain ratios $S_{\mathrm{r}}$ in plane bending.

and the number of cycles to failure $N_{\mathrm{f}}$ shown on the logarithmic graph are almost expressed by parallel straight lines for every strain ratio $S_{\mathrm{r}}$. Therefore, the relationships can be expressed by a power function similar to the CoffinManson law for low-cycle fatigue of normal metals as follows

$$
\varepsilon_{\max } \cdot N_{\mathrm{f}}^{\beta}=\alpha
$$

where $\alpha$ and $\beta$ represent $\varepsilon_{\max }$ in $N_{\mathrm{f}}=1$ and the slope of the $\log \varepsilon_{\max }-\log N_{\mathrm{f}}$ curve, respectively. Each curve was approximated. The average of approximated slope $\beta$ was 0.53 . Obtaining $\alpha$ using this average $\beta, \alpha$ can be approximated by the following functions of $S_{\mathrm{r}}$.

$$
\begin{array}{ll}
\alpha=1.2 S_{\mathrm{r}}+2.9 & ;-1 \leqq S_{\mathrm{r}} \leqq 0 \\
\alpha=5.33 S_{\mathrm{r}}+2.9 & ; S_{\mathrm{r}} \geqq 0
\end{array}
$$

The calculated values of eqs. (3) and (4) are shown by solid lines in Fig. 5. As can be seen, the overall inclinations are well approximated by the solid lines. However, the difference between the experimental data and the calculated values is large in the case of $S_{\mathrm{r}}=0$ and $S_{\mathrm{r}}=-1$.

In order to discuss the influence of strain ratio $S_{\mathrm{r}}$ on fatigue life, the stress-strain curves of the elements on the surface of the wires during cycling are schematically shown in Fig. 6. In Fig. 6, it is assumed that the stress-strain curve in the compression side is symmetric to that in the tension side. The area surrounded by the hysteresis loop in Fig. 6 denotes the

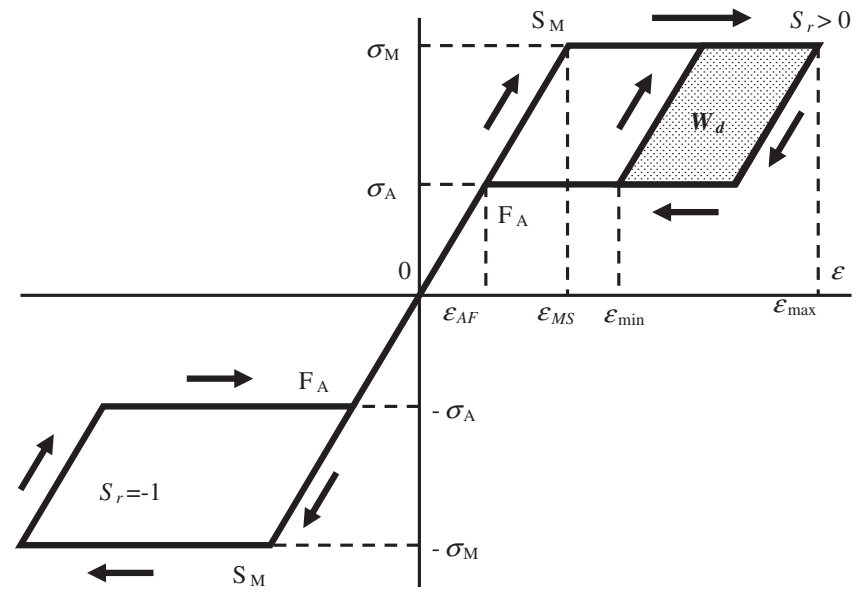

(a) SE-NT

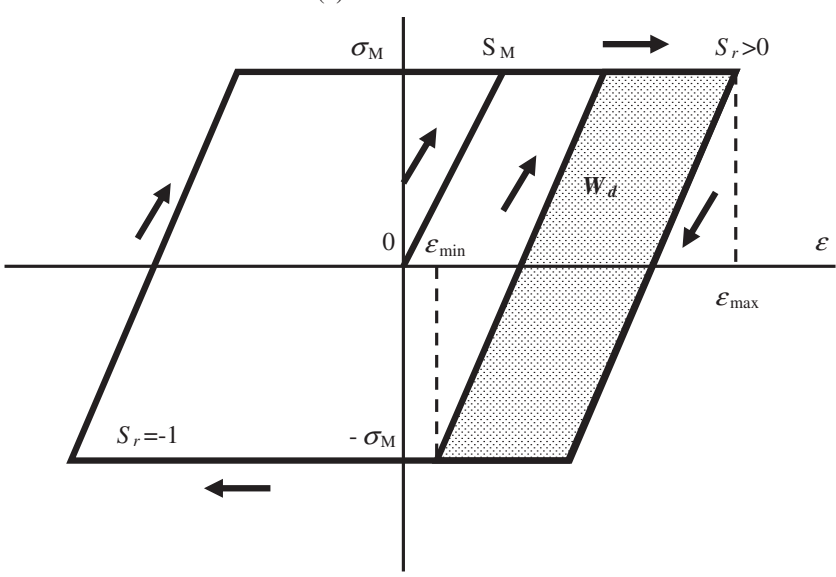

(b) SME-NT

Fig. 6 Schematic stress-strain diagrams during cycling for SE-NT and SME-NT (a) SE-NT, (b) SME-NT.

dissipated work $W_{\mathrm{d}}$ per unit volume. Since $S_{\mathrm{r}}=\varepsilon_{\min } / \varepsilon_{\max }$, the larger the $S_{\mathrm{r}}$ for the same $\varepsilon_{\max }$, the larger the $\varepsilon_{\min }$, resulting in smaller $W_{\mathrm{d}}$. If SMA is subjected to cyclic loading, temperature of the wire increases based on $W_{\mathrm{d}} \cdot{ }^{11)}$ The MT stress $\sigma_{\mathrm{M}}$ of SMA increases in proportion to an increase in temperature. ${ }^{14)}$ Therefore, as can be seen in Fig. 6(a), in the case of $S_{\mathrm{r}}>0$ for SE-NT, the smaller the $W_{\mathrm{d}}$, the smaller the fatigue damage due to small increase in $\sigma_{\mathrm{M}}$, resulting in longer life.

As can be seen in Fig. 5, in the case of $S_{\mathrm{r}}=0, N_{\mathrm{f}}$ for $\varepsilon_{\max }$ above $1.5 \%$ is almost the same as $N_{\mathrm{f}}$ for $S_{\mathrm{r}}=0.1$. This can be explained as follows. In the case of $S_{\mathrm{r}}=0.1, \varepsilon_{\min }$ is smaller than the strain $\varepsilon_{A F}$ at the reverse-transformation finish point $\mathrm{F}_{\mathrm{A}}$ and the reverse transformation completes. Therefore, the MT strain range $\Delta \varepsilon_{\mathrm{M}}=\varepsilon_{\max }-\varepsilon_{\mathrm{MS}}$ which represents the width of hysteresis loop of the stress-strain curve becomes equal for $S_{\mathrm{r}}=0$ and $S_{\mathrm{r}}=0.1$, resulting in the same value for $W_{\mathrm{d}}$. As a result, $N_{\mathrm{f}}$ for $S_{\mathrm{r}}=0$ coincides with $N_{\mathrm{f}}$ for $S_{\mathrm{r}}=0.1$.

In the case of alternating plane bending $\left(S_{\mathrm{r}}=-1\right), N_{\mathrm{f}}$ decreases markedly for large $\varepsilon_{\max }$. In this case, the surface element of the specimen is subjected to the MT by both tension and compression in each cycle. Therefore, $W_{\mathrm{d}}$ is twice, temperature increases correspondingly and fatigue damage is large according to an increase in the MT stress, resulting in short fatigue life. 


\subsection{Influence of strain ratio on fatigue life for SME-NT} and SE-NT

With respect to the plane bending fatigue for SME-NT in the range of strain ratio $S_{\mathrm{r}}$ from -1 to 0.3 , the fatigue-life curves are expressed by eq. (3) in which $\beta$ is constant while $\alpha$ increases monotonically in proportion to $S_{\mathrm{r}}{ }^{11)}$ This property of SME-NT is different from that of SE-NT in which $\beta$ is large for $S_{\mathrm{r}}=0$ and small for $S_{\mathrm{r}}=-1$ and $\alpha$ is expressed by eq. (4). The fatigue property for SME-NT appears according to the following reason. As can be seen from the stress-strain curves shown in Fig. 6(b), SME-NT is subjected to both the tensile and compressive MT during cyclic bending. The area surrounded by the hysteresis loop in each cycle $W_{\mathrm{d}}$ increases with decreasing $\varepsilon_{\min }$. Since $S_{\mathrm{r}}=\varepsilon_{\min } / \varepsilon_{\max }, W_{\mathrm{d}}$ decreases in proportion to $S_{\mathrm{r}}$ with respect to the same $\varepsilon_{\max }$. In this way, SME-NT is subjected to both the cyclic tensile and compressive MT and $W_{\mathrm{d}}$ decreases monotonically in proportion to $S_{\mathrm{r}}$, and therefore $\alpha$ increases monotonically in proportion to $S_{\mathrm{r}}$.

\subsection{Fatigue life of rotating bending and alternating plane bending for SE-NT}

Figure 7 shows the relationships between the bending strain amplitude on the surface of the wires $\varepsilon_{\mathrm{a}}$ and the number of cycles to failure $N_{\mathrm{f}}$ obtained by the rotating-bending and alternating-plane bending fatigue tests for SE-NT with frequency $f=500 \mathrm{cpm}$ at room temperature. In the figure, the points with arrows at $10^{7}$ cycles denote cases where failure did not occur. Strain ratio is -1 in both bending fatigue tests.

As can be seen, in the region of long fatigue life for $\varepsilon_{\mathrm{a}}$ smaller than $1.5 \%$, difference of fatigue life between rotating bending and alternating-plane bending is small. However, if $\varepsilon_{\mathrm{a}}$ is larger than $2 \%$, the fatigue life for the same $\varepsilon_{\mathrm{a}}$ in rotating bending become longer than that in alternating-plane bending. The overall fatigue life is similar to that of SME-NT. ${ }^{9)}$ In the case of alternating-plane bending, only the elements on the surface of the wire in both sides where maximum bending strain occurs are subjected to the cyclic MT. On the other hand, in the case of rotating bending, whole surface elements of the wire are subjected to the tensile and compressive MT in each cycle. If $\varepsilon_{\mathrm{a}}$ becomes large, temperature increases markedly due to the large dissipated work with the MT in the surface elements of the wire. Therefore, the MT stress in

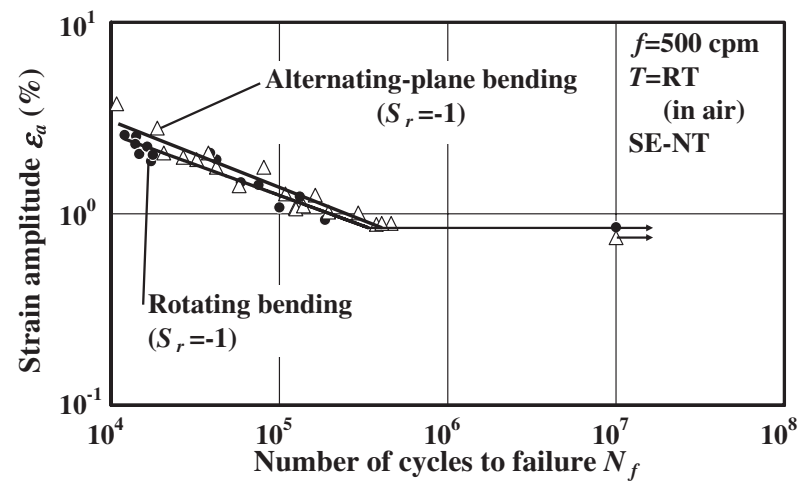

Fig. 7 Fatigue life curves in alternating-plane bending and rotating bending for SE-NT.

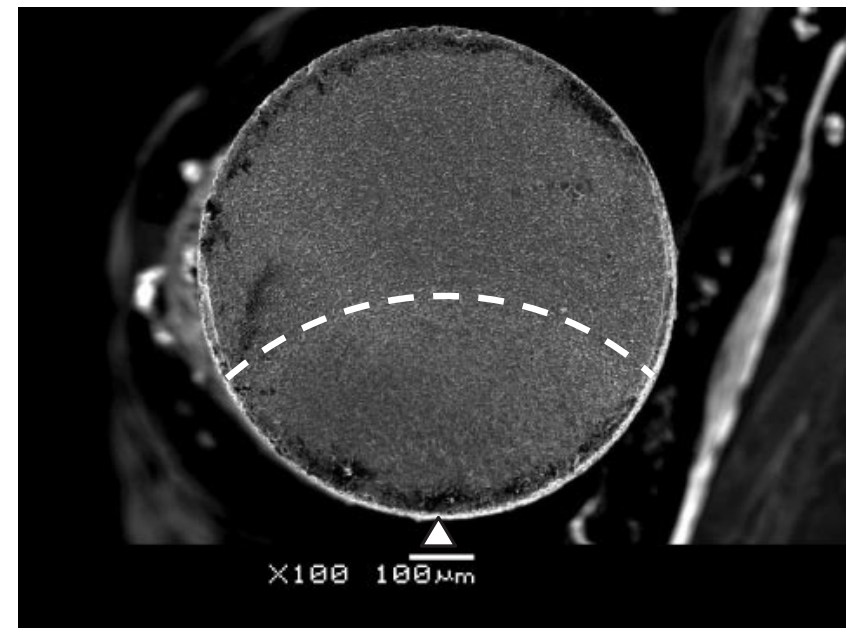

(a) Whole fracture surface

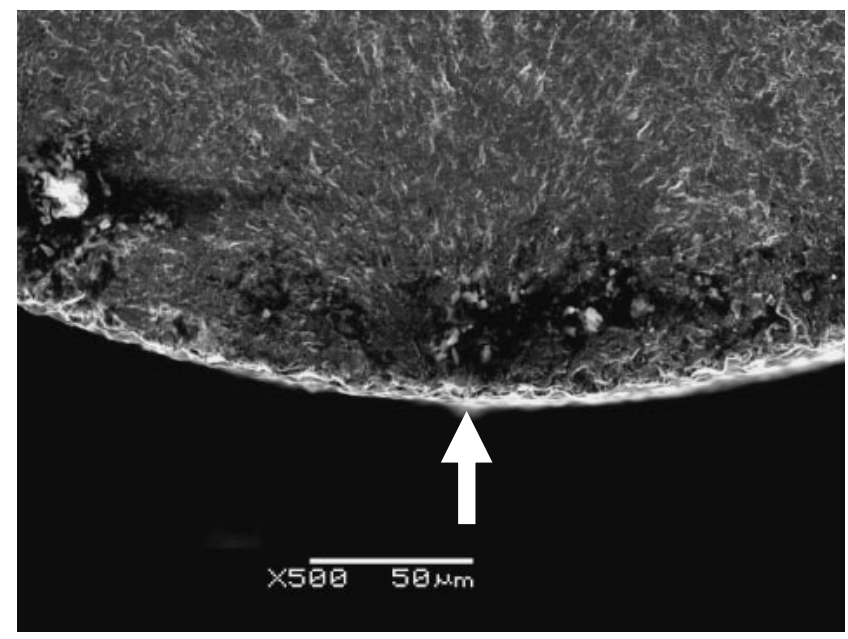

(b) Fracture surface at crack initiation

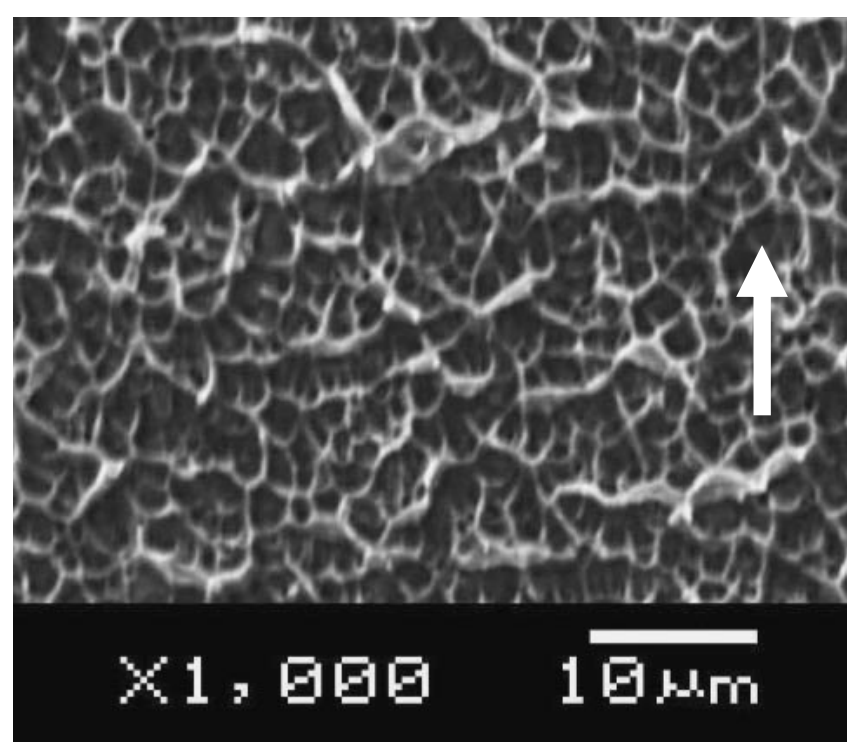

(c) Fracture surface of unstable fracture

Fig. 8 SEM photographs of fracture surface for SME-NT in alternatingplane bending. 
rotating bending is higher and fatigue damage is larger, resulting in shorter fatigue life.

\subsection{Observation of fatigue fracture surface}

Figure 8 shows SEM photographs of a fracture surface for SME-NT in the case of $f=500 \mathrm{cpm}, \varepsilon_{\mathrm{a}}=1.71 \%, N_{\mathrm{f}}=$ 11802 cycles at room temperature in alternating-plane bending. The whole fracture surface, the fracture surface at crack initiation and the fracture surface in the case of an unstable fracture are shown in Figs. 8(a), (b) and (c), respectively.

As can be seen in Figs. 8(a) and (b), the crack nucleates at a certain point on the surface of the wire and propagates towards the center in a sinuous radial pattern. Although small cracks are observed on the surface elements in both sides of the wire subjected to maximum bending strain, one single crack grows preferentially. Striation is not observed in the fatigue-crack growth region. Following the appearance of fatigue fracture with a fan-shaped surface, unstable fracture finally occurs. As can be seen in Fig. 8(c), isometric dimples with an average diameter of about $3 \mu \mathrm{m}$ appear in the region of the unstable fracture. In the case of SE-NT, the fatigue crack also nucleates on the surface of the wire, and a fanshaped fatigue-crack growth region and the unstable fracture region with isometric dimples are observed.

The phenomena that one single fatigue crack and a fanshaped fatigue crack region appear are similar to those observed for SMA wires subjected to rotating bending. ${ }^{13)}$ In the case of rotating-bending, though small cracks are observed on the whole surface of the wire, one single crack grows preferentially.

\subsection{Change in strain amplitude during fatigue test}

In the previous studies, bending strain of the specimen in the fatigue test was obtained as follows. The bent shape of the specimen was traced when the specimen was mounted on the experimental apparatus and the maximum bending strain on the surface of the specimen was determined by using the radius of curvature at a rupture part after fatigue fracture. Since temperature of the specimen increases during fatigue test, it is possible for the maximum strain to change. In order to examine the change in bending strain, the following test was carried out.

Temperature rise in rotating bending during fatigue test is larger than that in plane bending. 9) The larger the strain amplitude and the higher the frequency, the larger the temperature rise. Temperature increases markedly in the initial about $20 \mathrm{~s}$ and saturates in a certain value thereafter. Taking into account of these points, the rotating-bending fatigue test was carried out. Photograph of prescribed initial strain $\varepsilon_{\text {initial }}$ was taken by a digital camera. Photographs of the specimen during the rotating-bending fatigue test were taken at 50, 70, 80 and $90 \mathrm{~s}$ after the start of the test. Bending strain during rotation $\varepsilon_{\text {rotation }}$ was determined as an average value obtained from four images. The experiment was performed for SME-NT at frequencies $f=100,500$ and $1000 \mathrm{cpm}$ in air.

The relationships between $\varepsilon_{\text {initial }}$ and $\varepsilon_{\text {rotation }}$ are shown in Fig. 9. As can be seen, $\varepsilon_{\text {rotation }}$ is equal to $\varepsilon_{\text {initial }}$ when $\varepsilon_{\text {initial }}$ is smaller than $1 \%$. With $\varepsilon_{\text {initial }}$ larger than $1.2 \%, \varepsilon_{\text {rotation }}$

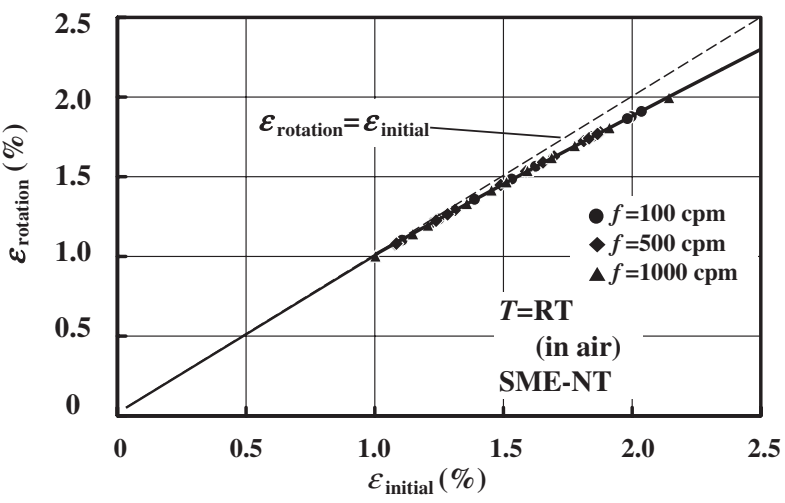

Fig. 9 Relationship between $\varepsilon_{\text {rotation }}$ and $\varepsilon_{\text {initial }}$ in rotating bending.

becomes gradually smaller than $\varepsilon_{\text {initial }}$. At $\varepsilon_{\text {initial }}=2 \%$, $\varepsilon_{\text {rotation }}$ is smaller than $\varepsilon_{\text {initial }}$ by $5 \%$ of $\varepsilon_{\text {initial }}$. These properties appear by the following reason. When strain exceeds $1 \%$, the MT occurs and temperature increases, resulting in an increase in the MT stress. Therefore, the central part of the specimen with maximum bending strain is forced to be recovered into a straight line. Based on this recovery force, $\varepsilon_{\text {rotation }}$ is relaxed. On the other hand, as can be seen in Fig. 9, the influence of $f$ on $\varepsilon_{\text {rotation }}$ is little.

\subsection{Discussion of fatigue crack growth based on meas- urement of electric resistance}

It is very difficult to observe directly the fatigue crack behavior in the thin SMA wire during bending fatigue test. In the present study, the fatigue crack behavior was discussed by considering the fact that electric resistance increases due to a decrease in cross-sectional area of the wire when fatigue crack grows. The fatigue crack growth was investigated by the alternating-plane bending fatigue test since the position of fatigue crack nucleation and the direction of crack propagation are definite. At first, an artificial notch with length $a_{\mathrm{m}}$ was made in a SME-NT wire model by a diamond cutter with a width of $0.5 \mathrm{~mm}$ and a diameter of knife edge circle of $157 \mathrm{~mm}$. Bottom of the notch is close to straight line (see Fig. 13). The relationship between notch length $a_{\mathrm{m}}$ and electric resistance $R$ was measured by setting a notched side of the model in tension and giving a prescribed bending strain.

Next, photographs were taken of fracture surface of the specimen obtained by the fatigue test. The relationship between notch length $a_{\mathrm{m}}$ of the model and fatigue crack length $a$ was obtained with respect to the same areas between the fatigue-crack propagated region and the notched part. Since an edge of the fatigue-crack propagated region is fanshaped, $a$ is larger than $a_{\mathrm{m}}$ by about $15 \%$. Figure 10 shows the relationship between $a$ and $R$ obtained by this way. In the figure, $d$ and $R_{0}$ denote diameter of the wire and electric resistance without crack, respectively. This relationship is a master curve to obtain $a$ by measuring $R$. In the alternatingplane bending test with $f=500 \mathrm{cpm}$, cyclic motion was stopped at the prescribed number of cycles and electric resistance $R$ was measured in the state of the notched side under maximum bending strain after $30 \mathrm{~s}$ from the stop. Crack length $a$ can be obtained corresponding to $R$ as shown in Fig. 10. 


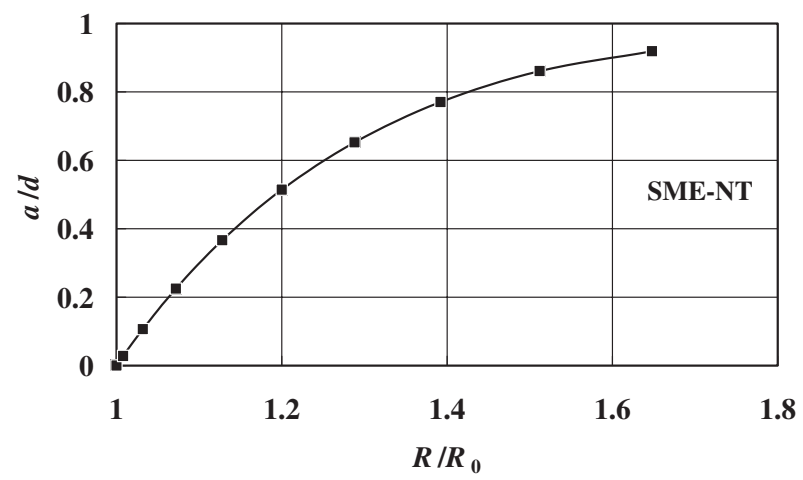

Fig. 10 Relationship between fatigue crack length $a$ and electric resistance $R$.

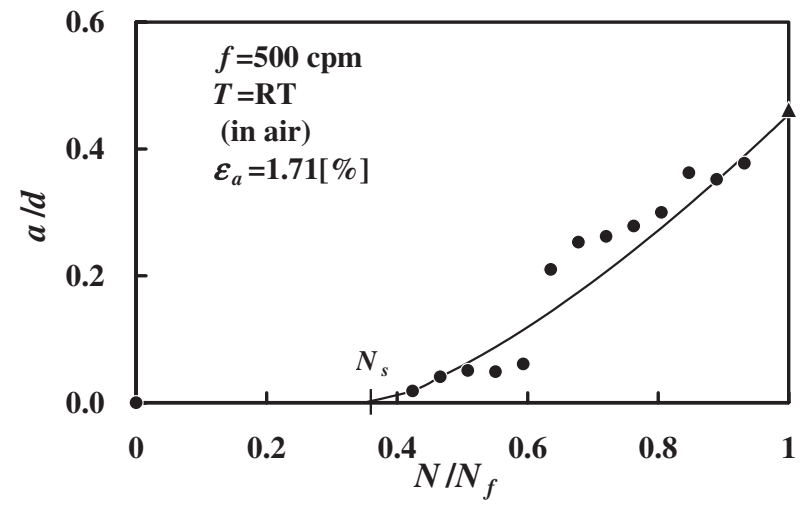

Fig. 11 Relationship between fatigue crack length $a$ and the number of cycles $N$ obtained by measuring electric resistance.

Figure 11 shows the relationship between $a$ and the number of cycles $N$ for strain amplitude $\varepsilon_{\mathrm{a}}=1.71 \%$. In the figure, $N_{\mathrm{f}}$ denotes the number of cycles to failure. The value of $a$ at $N=N_{\mathrm{f}}$ is obtained from the fatigue fracture surface. $\mathrm{A}_{\mathrm{S}}$ can be seen, fatigue crack starts to grow from the number of cycles $N_{\mathrm{s}}=0.35 N_{\mathrm{f}}$ and grows markedly thereafter till final rupture. It is confirmed that fatigue crack growth can be obtained by measuring electric resistance. In order to improve the accuracy, it is necessary to make the width of artificial notch narrow, to make the bottom of the notch fanshaped and to improve electrode for measuring electric resistance.

\subsection{Fatigue crack growth for the notched material}

In the previous section 3.7, fatigue crack growth was discussed from the view point of the change in electric resistance. In this section, the fatigue crack behavior is discussed based on the observation of fracture surface. In order to obtain the fracture surface, the notched specimen was removed from the fatigue test machine at the prescribed number of cycles during alternating-plane bending fatigue test and was forced to rupture through a tension machine.

In the specimen of SME-NT, the notch with length $a_{0}=0.1 \mathrm{~mm}$ was made using the same diamond cutter as explained in section 3.7. Figure 12 shows the relationships between strain amplitude $\varepsilon_{\mathrm{a}}$ and the number of cycles to failure $N_{\mathrm{f}}$ obtained by the alternating-plane bending fatigue

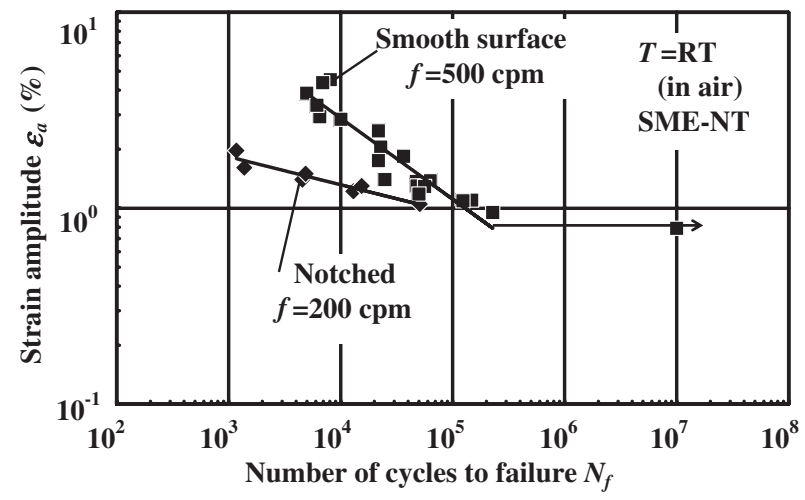

Fig. 12 Fatigue life curves for notched and smooth surface specimens of SME-NT in alternating-plane bending.

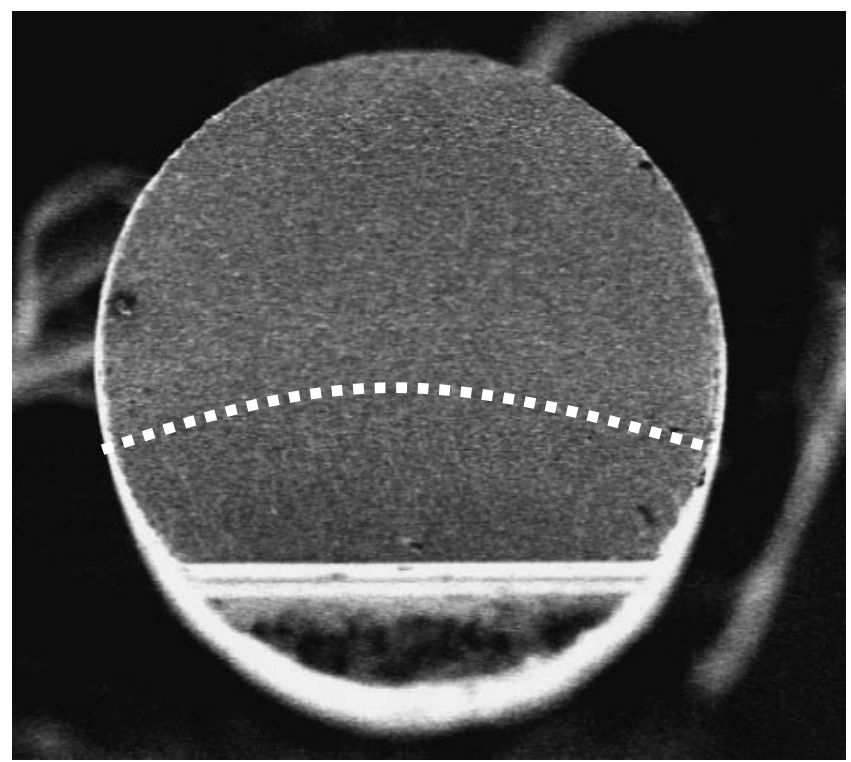

Fig. 13 Surface ruptured by forced-tension during fatigue crack growth for notched specimen at $N=5655$ cycles.

test for the specimen with and without notch. In the case of the specimen without notch, the higher the frequency $f$, the shorter the fatigue life $N_{\mathrm{f}}{ }^{8)}$ As can be seen in Fig. $12, N_{\mathrm{f}}$ for notched specimen is short even if $f$ is low. The larger the $\varepsilon_{\mathrm{a}}$, the shorter the $N_{\mathrm{f}}$ compared to $N_{\mathrm{f}}$ for the specimen without notch.

Figure 13 shows a photograph of the fracture surface of specimen which was obtained by forced-tension to measure crack length $a$ during fatigue test. This shows the fracture surface in the case of $\varepsilon_{\mathrm{a}}=1.6 \%$ and the number of cycles $N=5655$. As can be seen, fatigue crack with a fan-shaped front propagates from the bottom of notch. Fatigue crack length $a$ from the surface of the wire is obtained based on this photograph. Figure 14 shows the relationships between $a$ and the number of cycles $N$ obtained by this method. In the experiments, the specimens were ruptured at the prescribed $N$ for various $\varepsilon_{\mathrm{a}}$ to obtain $a$. In the figure, $a$ at $N=N_{\mathrm{f}}$ denotes the crack length obtained by the fatigue test without forcedtension. As can be seen in Fig. 14, fatigue crack grows with an increase in $N$ and grows markedly just before failure. The reason why definite difference was not observed in fatigue 


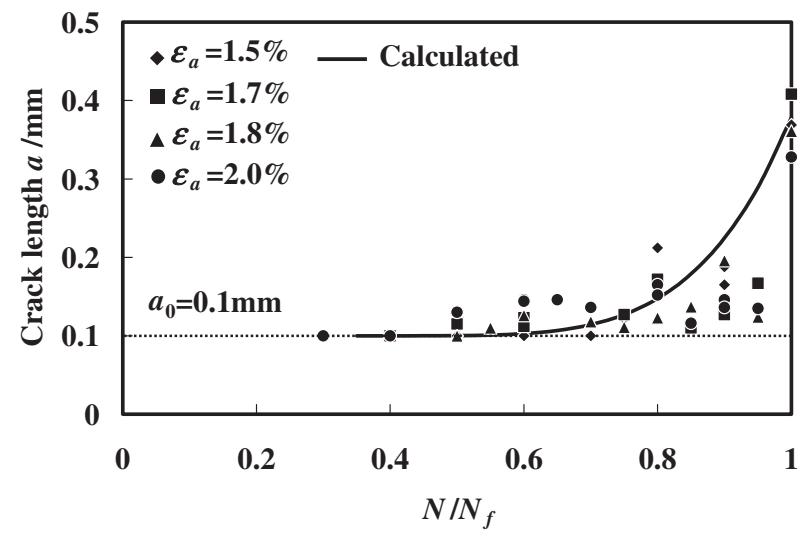

Fig. 14 Relationship between crack length $a$ and the number of cycles obtained by forced-tension rupture during fatigue crack growth.

growth behavior for various strain amplitudes $\varepsilon_{\mathrm{a}}$ may be considered due to the accuracy of making the notch. That is, the magnitude of $a_{0}$ and the bottom shape of the notch could not be made exactly the same in all specimen. The relationship between $a$ and $N$ is approximated by the following equation

$$
a-a_{0}=A\left(\frac{N-N_{\mathrm{s}}}{N_{\mathrm{f}}}\right)^{B}
$$

where $a_{0}=0.1 \mathrm{~mm}$ for notch length and $N_{\mathrm{s}}$ denotes the number of cycles for crack nucleation. The calculated result is shown in Fig. 14 by the solid line with $A=2.2 \mathrm{~mm}, B=$ 4.8 and $N_{\mathrm{s}}=0.35 N_{\mathrm{f}}$. As can be seen, the inclination of fatigue crack growth is well expressed by eq. (5).

\section{Conclusions}

The influence of strain ratio on bending fatigue properties and fatigue crack growth in TiNi SMA wires were investigated. The results obtained are summarized as follows.

(1) The MT stress of the superelastic thin wire (SE-NT) is higher than that of the SMA thin wire (SME-NT) and the fatigue life of SE-NT is shorter than that of SMENT. Maximum bending strain at the fatigue limit is the MT starting strain.

(2) The low-cycle fatigue life curve in plane bending for SE-NT is expressed by a power function of maximum strain $\varepsilon_{\max }$ and the number of cycles to failure $N_{\mathrm{f}}$. The smaller the strain ratio for the same $\varepsilon_{\max }$, the shorter the fatigue life.

(3) In both the rotating bending and the plane bending, fatigue cracks nucleate on the surface of the wire and one fatigue crack grows preferentially. The region in which fatigue crack propagated is fan-shaped.
(4) Maximum bending strain $\varepsilon_{\max }$ does not change during the rotating-bending fatigue test if an initially designated $\varepsilon_{\max }$ is smaller than $1 \%$ but decreases gradually if an initial $\varepsilon_{\max }$ is larger than $1 \%$. In the case of $2 \%$ of an initial $\varepsilon_{\max }, \varepsilon_{\max }$ decreases by $5 \%$ of the initial $\varepsilon_{\max }$ during the fatigue test.

(5) If fatigue crack grows, cross-sectional area at the crackpropagated part decreases. Fatigue crack growth can be estimated by measuring the change in electric resistance.

(6) The fatigue crack growth of the notched specimen was investigated by forced-tension rupture in the process of fatigue test. The fatigue crack length is expressed by a power function of the number of cycles.

\section{Acknowledgement}

The experimental work of this study was carried out with the assistance of students in Aichi Institute of Technology, to whom the authors wish to express their gratitude. The authors also wish to extend their thanks to the Japan Society for Promotion of Science for the provision of the Basic Research (C) of a Grant-in-aid of Scientific Research.

\section{REFERENCES}

1) H. Funakubo, ed.: Shape Memory Alloys, (Gordon and Breach Science Pub. 1987) pp. 1-60.

2) T. W. Duering, K. N. Melton, D. Stockel and C. M. Wayman, eds.: Engineering Aspects of Shape Memory Alloy, (Butterwoth-Heinemann, 1990) pp. 1-35.

3) K. Otsuka and C. M. Wayman, eds.: Shape Memory Materials, (Cambridge University Press, 1998) pp. 1-49.

4) T. Saburi, ed.: Shape Memory Materials, (Trans Tech Pub, 2000) pp. 315-366.

5) Y. Y. Chu and L. C. Zhao, eds.: Shape Memory Materials and Its Applications, (Trans Tech Pub, 2002) pp. 177-284.

6) T. Sakuma, U. Iwata and Y. Kimura: Cyclic Behavior and Fatigue Life of TiNiCu Shape Memory Alloy. Fatigue '96 1 (1996) 173-178.

7) R. L. Holtz, K. Sadananda and M. A. Imam: Int. J. Fatigue 21 (1999) 137-145.

8) A. L. McKelvey and R. O. Ritchie: Metall. Mater. Trans. A 32A (1999) 731-743.

9) H. Tobushi, T. Hachisuka, T. Hashimoto and S. Yamada: Trans. ASME, J. Eng. Mater. Tech. 120 (1998) 64-70.

10) H. Tobushi, K. Okumura, K. Nakagawa and K. Takata: Trans. Mater. Res. Soc. Jpn. 26 (2001) 347-350.

11) Y. Furuichi, H. Tobushi, T. Ikawa and R. Matsui: Proc. Instn. Mech. Engrs., Vol. 217, Part L: J. Maters.: Design and Appl. (2003) 93-99.

12) T. Swaguchi, G. Kaustrater, A. Yawny, M. Wagner and G. Eggeler: Metall. Mater. Trans. A 34A (2003) 2847-2860.

13) R. Matsui, H. Tobushi, Y. Furuichi and H. Horikawa: Trans. ASME, J. Eng. Mater. Tech. 126 (2004) 384-391.

14) J. A. Shaw and S. Kyriakides: J. Mech. Phys. Solids 43 (1995) $1243-$ 1281. 